\title{
Mortality of adult intensive care units in Turkey using the APACHE II and SOFA systems (outcome assessment in Turkish intensive care units)
}

Cetin Kaymak ${ }^{1}$, Irfan Sencan², Seval Izdes ${ }^{3}$, Aydin Sari ${ }^{4}$, Hatice Yagmurdur ${ }^{5}$, Derya Karadas ${ }^{4}$, Derya Oztuna ${ }^{6}$

\author{
${ }^{1}$ Anesthesiology and Reanimation Department, Intensive Care Unit, Ankara Training \\ and Research Hospital, Ankara, Turkey \\ ${ }^{2}$ General Directorate of Health Services, Ministry of Health, Ankara, Turkey \\ ${ }^{3}$ Anesthesiology and Reanimation Department, Intensive Care Unit, Faculty \\ of Medicine, University of Yildirim Beyazit, Ankara, Turkey \\ ${ }^{4}$ Directorate of Audit Department, Ministry of Health, Ankara, Turkey \\ ${ }^{5}$ Anesthesiology and Reanimation Department, Intensive Care Clinic, Numune Training \\ and Research Hospital, Ankara, Turkey \\ ${ }^{6}$ Medical Biostatistics Department, Faculty of Medicine, University of Ankara, Ankara, \\ Turkey
}

Submitted: 27 October 2015

Accepted: 28 March 2016

Arch Med Sci 2018; 14, 3: 510-515

DOI: https://doi.org/10.5114/aoms.2016.59709

Copyright (c) 2016 Termedia \& Banach

\section{Abstract}

Introduction: The aim of this study was to evaluate intensive care unit (ICU) performance using risk-adjusted ICU mortality rates nationally, assessing patients who died or had been discharged from the ICU. For this purpose, this study analyzed the Acute Physiology and Chronic Health Evaluation (APACHE) II and Sequential Organ Failure Assessment (SOFA) databases, containing detailed clinical and physiological information and mortality of mixed critically ill patients in a medical ICU at secondary and tertiary referral ICUs in Turkey.

Material and methods: A total of 690 adult intensive care units in Turkey were included in the study. Among 690 ICUs evaluated, $39.7 \%$ were secondary and $60.3 \%$ were tertiary ICUs. A total of 4188 patients were enrolled in this study. Intensive care units of ministry, university, and private hospitals were evaluated all over Turkey. During the study period, clinical data that were collected concurrently for each patient contained demographic details and the diagnostic category leading to ICU admission. APACHE II and SOFA scores following ICU admission were calculated and recorded. Patients were followed up for outcome data until death or ICU discharge.

Results: The mean age of patients was $68.8 \pm 19$ and $54 \%$ of them were male. The mean APACHE II score was $20 \pm 8.7$. The ICUs' mortality rate was $46.3 \%$, and mean predicted mortality was $37.2 \%$ for APACHE II. The standardized mortality ratio was 1.28 (95\% confidence interval: 1.21-1.31).

Conclusions: There was a wide difference in outcome for patients admitted to different ICUs and severity of illness using risk adjustment methods. The high mortality rate in patients could be related to comorbid diseases, high mechanical ventilation rates and older ages.

Key words: critical care, severity of illness, mortality, survival.

\author{
Corresponding author: \\ Cetin Kaymak MD, PhD, \\ Assoc Prof. \\ Ministry of Health \\ Ankara Training and Research \\ Hospital \\ Anesthesiology \\ and Reanimation Department \\ Intensive Care Unit \\ Ulucanlar, Altındağ \\ 06330, Ankara, Turkey \\ Phone: +90 3125953172 , \\ +905326545426 \\ Fax: +90 3123633330 \\ E-mail: cetinkaymak@ \\ yahoo.com
}




\section{Introduction}

The outcome in critically ill patients concerned with prognosis has many background effects of risk factors such as age, gender, severity of illness, comorbidities, diagnosis, and response to therapy. An aging population and chronic diseases may also result in an increased number of deaths in intensive care unit (ICU) patients [1-3]. Clinical results have increased the need for outcome examination and guidance on effective use. For this reason, there is an increasing demand for critical care in the population at risk [4-7].

The scoring systems could be used to predict the expected mortality rate that is adjusted for differences in diagnosis, physiologic abnormalities, and outcome of critically ill patients admitted to an ICU [6, 8-11]. For this reason, general severity of illness scoring systems are becoming popular and allow one to make international comparisons of intensive care outcome. Although there are difficulties in using risk adjustment methods to compare outcome for ICUs, several studies have reported a beneficial performance of Acute Physiology and Chronic Health Evaluation II (APACHE II) $[6,10,12-14]$ and Sequential Organ Failure Assessment (SOFA) [5-7, 10, 15-17] scores in predicting the actual or standardized mortality ratio (SMR) in individual ICUs. Although organ dysfunction scores were not developed for estimating mortality, a meaningful correlation can be shown between combined organ dysfunction scores and degree of organ failure and mortality $[5,15,18]$. Still, severity of illness and actual or SMR are being used by different institutions to assess the clinical performance and quality of care in an ICU $[11,19,20]$. The aim of this study was to evaluate risk-adjusted mortality among patients who died or were discharged from Turkish ICUs. Regarding this, the present study analyzed APACHE II and SOFA databases in critically ill patients at secondary and tertiary referral hospital ICUs in Turkey.

\section{Material and methods}

This study consisted of adult patients who were admitted to the ICU to receive ICU-specific intervention or physiological monitoring without receiving an ICU-specific intervention and was conducted in a total of 690 medical, surgical or mixed ICUs. The ICUs were evaluated by teams composed of 330 medical staff who had undergone intensive care training.

Patients were excluded if they were younger than 18 years old or burn patients, or had a length of ICU stay of less than $24 \mathrm{~h}$, or had undergone coronary artery bypass grafting, as they have different risk-adjustment profiles than other critically ill patients. This study was approved by the
Institutional Ethics Committee. After approval of the study, the clinical database of ICUs was linked to a data system formed by the Audit Department of the Ministry of Health. This system contained data for all public and private hospital admissions in Turkey. During the research period, data integrity was maintained by having a single data custodian, who checked all data entered and resolved data inconsistencies.

Data collection took place from 1 June to 30 June 2012. During the study period, clinical data that were collected concurrently for each patient contained demographic details, diagnostic category leading to ICU admission and location before ICU admission (emergency, hospital, or operating room; transfer from another ICU or hospital). Data were collected for each patient admitted to the ICU and the patients were followed for 28 days. Reasons for ICU admission were divided into 10 diagnostic categories: respiratory, cardiovascular, neurologic, gastrointestinal, renal-metabolic, infection, hematologic-oncologic, trauma, intoxication, and postoperative. We categorized duration of ICU stay into below and above $48 \mathrm{~h}$ and then patients staying longer than $48 \mathrm{~h}$ were again divided into two groups: below and above 15 days. APACHE II and SOFA scores during the first $24 \mathrm{~h}$ following ICU admission were calculated and recorded. Patients were followed up for outcome data until death or ICU discharge. The prediction of risk of ICU death was performed with the original equation and coefficients described by Knaus et al. $[8,9]$. The $95 \%$ confidence intervals $(\mathrm{Cl})$ were calculated for the mortality ratio (observed mortality/predicted mortality). The observed mortality is regarded as a Poisson variable, and its $95 \% \mathrm{Cl}$ is divided by the predicted mortality to derive the $95 \%$ confidence interval of the mortality ratio. In addition, we also determined whether or not a patient was receiving invasive mechanical ventilation, or had central venous catheterization. The predicted hospital mortality rates were calculated using the logistic regression model. The equation coefficients for APACHE II were supplied by APACHE Medical Systems. The mortality in intensive care units was analyzed in subgroups as APACHE II and SOFA scores. The mortality in subgroups was compared to mortalities reported in the literature.

\section{Statistical analysis}

Statistical analyses were performed using SPSS 11.5. As descriptive statistics, mean \pm standard deviation (SD) [median (min.-max.)] or frequency (percent) was given. To compare independent groups, for categorical variables the $\chi^{2}$ test and for metric variables the Mann-Whitney $U$ test were used. $P$-value $<0.05$ was considered as statistically significant. 


\section{Results}

In this study, a total of 690 ICUs of ministry, university and private hospitals all over Turkey were evaluated. Among 690 ICUs evaluated, $39.7 \%$ were secondary and $60.3 \%$ were tertiary ICU. The ICUs represented 2422 beds and 3702 beds of secondary and tertiary ICUs, respectively. The percentages of beds in public, university and private hospitals in Turkey were $47.2 \%, 21.8 \%$, and $30.9 \%$, respectively. One hundred and thirtysix (19.7\%) ICUs were medical, 105 (15.2\%) were surgical, and 449 (65.1\%) were combined medical-surgical.

From 690 ICUs, a total of 4188 patients were enrolled. Approximately $54 \%$ (53.7\%) of patients were male. The mean \pm SD age of the patients was $65.88 \pm 19.05$ years, and $69 \%$ were $>60$ years. The admissions of patients to the ICUs were $33.5 \%$ from emergency departments, $23.3 \%$ from clinics in hospitals, $7.7 \%$ from operating rooms, $4.8 \%$ from home, $21.9 \%$ from another hospital, $2.8 \%$ from secondary ICUs, $2.1 \%$ from primary ICUs, $2 \%$ from tertiary ICUs and $1.9 \%$ from the event scene. The most frequent reasons for ICU admission were neurologic disorders (35.9\%) and respiratory disorders (17.3\%). When patients over 60 years old were evaluated according to diagnosis, the percentage of respiratory disorders was $19.9 \%$ and that of neurologic disorders was $37.5 \%$.

The mean APACHE II and SOFA scores were 20 \pm 8.8 and $6.1 \pm 3.8$ respectively. The distribution of the APACHE II and SOFA scores for each diagnostic category is shown in Table I. The mechanical ventilation was determined as the most frequently performed attempt, with a percentage of $65.7 \%$ in ICUs. Mortality according to diagnostic categories varied widely from $0 \%$ to $61.8 \%$ (Table I). The ratio of central venous catheterization was $56 \%$. It was followed by antibiotic administration and inotropic agent administration for hemodynamic stabilization with rates of $78.7 \%$ and $23.3 \%$, respectively.

The mean length of ICU stay was $21.1 \pm 44.7$ days. Whereas the mean length of ICU stay was $21.8 \pm 35.7$ days for non-survivors, it was $21 \pm 51.8$ days for survivors. When the duration of ICU stay was categorized as above or below 15 days, it was found that $31.6 \%$ of patients stayed longer than 15 days. Among the patients who stayed longer than 15 days, $54.2 \%$ were male. In these patients, the rates of the need for mechanical ventilation, central venous catheterization and infection were $70.5 \%, 72.7 \%$, and 70.3 respectively. The mean \pm SD APACHE II score was $22.3 \pm 8.3$ for patients who stayed longer than 15 days and $19.2 \pm 8.8$ for patients who stayed shorter than 15 days. The distribution of the APACHE II and SOFA scores for age groups and staying above or below 15 days in the ICU is given in Table II. Also, the ICU stay according to admission of patients to ICUs was classified as within $48 \mathrm{~h}$ or after $48 \mathrm{~h}$ (Table II). In addition, it was followed by antibiotic administration and inotropic agent administration for hemodynamic stabilization with rates of $77.6 \%$ and $25.1 \%$, respectively. $3.6 \%$ of ICU patients were chronic haemodialysis patients, and it was found that $7.6 \%$ of

Table I. Distribution of APACHE II and SOFA scores and mortality rate for each diagnostic category

\begin{tabular}{|c|c|c|c|c|c|c|c|c|c|c|c|}
\hline \multirow[t]{2}{*}{ System } & \multirow[t]{2}{*}{ Score } & \multicolumn{10}{|c|}{ Diagnostic categories } \\
\hline & & I & II & III & IV & V & VI & VII & VIII & IX & $\mathrm{X}$ \\
\hline \multirow[t]{5}{*}{ APACHE II (\%) } & $0-10$ & 9.2 & 6.3 & 12 & 23.6 & 6.3 & 7.8 & 13.4 & 22.3 & 48.6 & 79.3 \\
\hline & $11-20$ & 44.8 & 39.4 & 40 & 42.4 & 37.1 & 44.8 & 44.4 & 48.3 & 27.8 & 10.3 \\
\hline & $21-30$ & 33.8 & 40.4 & 37.6 & 26.1 & 43.3 & 34.6 & 30.5 & 23.1 & 19.4 & 10.3 \\
\hline & $31-40$ & 10.3 & 11.5 & 8.6 & 6.4 & 11.2 & 10.8 & 9.6 & 5 & 4.2 & - \\
\hline & $>40$ & 2 & 2.3 & 1.8 & 1.5 & 2.2 & 2 & 2.1 & 1.2 & - & - \\
\hline \multirow[t]{5}{*}{ SOFA (\%) } & $0-5$ & 48.3 & 41.6 & 45.9 & 51.1 & 46.1 & 35.4 & 39.2 & 49.5 & 55 & 78.6 \\
\hline & $6-10$ & 41.3 & 39 & 41.8 & 35.1 & 32.4 & 42.5 & 37.3 & 37.4 & 36.7 & 14.3 \\
\hline & $11-15$ & 9.6 & 18.1 & 10.9 & 10.1 & 18.7 & 19.4 & 18.4 & 11.7 & 6.7 & - \\
\hline & $16-20$ & 0.8 & 1 & 1.3 & 3.2 & 2.3 & 2 & 4.1 & 1.4 & 1.7 & - \\
\hline & $>20$ & - & 0.3 & 0.1 & 0.5 & 0.5 & 0.7 & 0.9 & - & - & 7.1 \\
\hline \multirow[t]{2}{*}{ (\%) } & MVR & 69.5 & 69.6 & 65.1 & 52.1 & 56.4 & 66.6 & 64 & 68.2 & 74 & 80.6 \\
\hline & $M R$ & 46.7 & 50.9 & 47.4 & 41.2 & 55.8 & 49.7 & 61.8 & 26.6 & 11 & 25.1 \\
\hline
\end{tabular}

Cells represent column percentages: I - respiratory, II - cardiovascular, III - neurologic, IV - gastrointestinal, V - renal-metabolic, $V I$ - infection, VII - hematologic-oncologic, VIII - trauma, IX - intoxication, X - postoperative, MVR - mechanical ventilation rate, $M R$ - mortality rate. 
Table II. Distribution of the APACHE II and SOFA scores for age groups, short and long stay, and all of the deceased in the intensive care unit

\begin{tabular}{|c|c|c|c|c|c|c|c|}
\hline \multicolumn{2}{|c|}{ APACHE Score } & \multicolumn{5}{|c|}{ APACHE (\%) } & \multirow[t]{2}{*}{ Mean \pm SD APACHE } \\
\hline & & $0-10$ & $11-20$ & $21-30$ & $31-40$ & $>40$ & \\
\hline \multirow[t]{2}{*}{ Age groups } & $\leq 60$ years & 25.4 & 42.8 & 26.1 & 4.7 & 1 & $22.2 \pm 8.2$ \\
\hline & $>60$ years & 7.3 & 40.9 & 38.5 & 11 & 2.3 & $18.4 \pm 7.1$ \\
\hline \multirow[t]{2}{*}{ Short stay } & $\leq 48 \mathrm{~h}$ & 95.2 & 90.5 & 85.8 & 87.1 & 88.6 & $19.8 \pm 8.8$ \\
\hline & $>48 \mathrm{~h}$ & 4.8 & 9.5 & 14.2 & 12.9 & 11.4 & $22.4 \pm 7.9$ \\
\hline \multirow[t]{2}{*}{ Long stay } & $\leq 15$ days & 82.5 & 68.9 & 61.5 & 56.8 & 58.6 & $19.2 \pm 8.8$ \\
\hline & $>15$ days & 17.5 & 31.1 & 38.5 & 43.2 & 41.4 & $22.3 \pm 8.3$ \\
\hline \multicolumn{2}{|c|}{ All of the deceased } & 19.3 & 44.7 & 55.7 & 63.4 & 57.1 & $22.4 \pm 8.1$ \\
\hline \multirow{2}{*}{\multicolumn{2}{|c|}{ SOFA Score }} & \multicolumn{5}{|c|}{ SOFA (\%) } & Mean \pm SD SOFA \\
\hline & & $0-5$ & $6-10$ & $11-15$ & $16-20$ & $>20$ & \\
\hline \multirow[t]{2}{*}{ Age groups } & $\leq 60$ years & 47.6 & 38.6 & 12 & 1.5 & 0.2 & $5.8 \pm 3.7$ \\
\hline & $>60$ years & 44.3 & 40.2 & 13.5 & 1.6 & 0.3 & $6.2 \pm 3.8$ \\
\hline \multirow[t]{2}{*}{ Short stay } & $\leq 48 \mathrm{~h}$ & 89.5 & 86.9 & 90.6 & 98 & 98 & $6.1 \pm 3.8$ \\
\hline & $>48 \mathrm{~h}$ & 10.5 & 13.1 & 9.4 & 0.2 & 0.2 & $6.1 \pm 3.1$ \\
\hline \multirow[t]{2}{*}{ Long stay } & $\leq 15$ days & 68.5 & 63.6 & 61.9 & 83.9 & 100 & $6 \pm 3.9$ \\
\hline & $>15$ days & 31.5 & 36.4 & 38.1 & 16.1 & 0 & $6.3 \pm 3.5$ \\
\hline \multicolumn{2}{|c|}{ All of the deceased } & 39.6 & 50 & 69.8 & 67.3 & 44.4 & $6.9 \pm 4$ \\
\hline
\end{tabular}

all patients were treated with renal replacement therapies (RRT) in the ICU.

The ICUs' mortality rate was $46.3 \%$, and mean predicted mortality was $37.2 \%$ for APACHE II. The SMR was 1.28 (95\% Cl: 1.21-1.31). In all of the deceased, $63.8 \%$ were in tertiary ICUs, whereas $36.2 \%$ were in secondary ICUs. The APACHE II and SOFA scores in all of the deceased are given in Table II. The mortality rate of patients who stayed more than 15 days in the ICU was $52.4 \%$. The mortality rates of patients admitted to the ICU in the first $48 \mathrm{~h}$ and after $48 \mathrm{~h}$ were $46 \%$ and $49 \%$, respectively. In order to predict mortality, the logistic regression model was constructed with the APACHE-II score as an independent variable. The APACHE-II scorer was statistically significant $(\mathrm{OR}=1.059 ; 95 \% \mathrm{Cl}$ : 1.050-1.068).

In all of the deceased, the mean APACHE II and SOFA scores were $22.4 \pm 8.1$ and $6.9 \pm 4$ respectively. The mean APACHE II score was higher for non-survivors. In all of the deceased, the mean APACHE II score was $22.8 \pm 8.1$ for the patients who stayed longer than 15 days and $22.2 \pm 8$ for patients who stayed shorter than 15 days.

\section{Discussion}

In the present study, the patients hospitalized in 690 ICUs of ministry, university, and private hospitals were analyzed all over Turkey. This study was a large assessment of ICUs in institutions in Turkey. The present study revealed that the overall mortality was $46.3 \%$ and SMR was 1.28 . Our results showed that the higher observed mortality rate compared with predicted mortality rate corrected for severity of illness in patients was different in diagnostic categories of patients admitted to ICUs. The percentage of non-university type of hospital such as public was $47.2 \%$ and of university was $21.8 \%$, whereas that of non-university was $33.3 \%$ and university $57.4 \%$ in the ICON study [21].

More demographic data of the patients have been reported in the publications of APACHE II. In previous studies, the overall ICU mortality has been reported between $9 \%$ and $41 \%[1,2,14$, 21-25]. Although there may be a limitation in the ability of APACHE II to identify differences between ICUs, these differences may be related to the structure and process of the delivery of care between ICUs. The major limitation of this scoring system is that in many patients, despite the presence of comorbid conditions, selection of a principal diagnosis category may be difficult. In addition, the physiological variables, resuscitation and treatment are dynamic factors and can be influenced by multiple parameters. The authors in many studies have reported the considerable effect of small changes in the APACHE II score on predicted mortality, and this effect was marked for patients with a low predicted mortality at admission [12, 13, 26-29]. Markgraf et al. [14] showed that customization of APACHE II and III in a large patient population from a single unit led to an improvement in the overall. Despite a similar improvement of fit in several subgroups that were 
large enough to be tested, good uniformity of fit was not achieved. In addition, in that study the observed mortality rate was considerably higher than the predicted mortality for APACHE II and APACHE III models. We found that deceased with an ICU admission had a higher degree of chronic illness such as hematologic-oncologic, renal and cardiovascular diseases. One study conducted in Turkey that enrolled 334 ICU patients reported a risk of death of $46.7 \%$. The high mortality rate was associated with the patient population and nosocomial infections [23]. In the Intensive Care Over Nations Study, the ICU mortality rate was between $22.4 \%$ and $35.3 \%$ in the whole population [21]. Whereas the mean APACHE II score was $17.9 \pm 9.4$ in the ICON study, the mean APACHE II score was $20 \pm 8.8$ in our results. The mean APACHE II score was $22.4 \pm 8.1$ in non-surviving patients, whereas it was $18.3 \pm 8.9$ in surviving patients. In the reports from other countries, mortality rates of $24-41 \%$ and SMR of 0.91-1.34 were reported from different ICUs [1]. Hantke et al. [18] evaluated SOFA and APACHE II scores of 874 surgical intensive care patients, and assessed mortality curves. They found AUC values for APACHE II and SOFA of 0.73 and 0.71 , respectively. The study demonstrated a clear correlation between an elevated SOFA score and the mortality of the patients during their ICU stay. Another study that assessed SOFA score over the first 5 days of admission instead of over the entire ICU stay found an AUC of 0.79, which was almost the same as the AUC for a single SOFA score at admission [15].

In critically ill patients, the effects of mortality related to length of stay remain controversial due to differences in case-mix and definition of a prolonged stay in the ICU, but the length of stay in the ICU can influence the observed mortality rate and increase the SMR. In our study, when the patients who stayed longer than 15 days in ICUs were analyzed, there was no difference between non-survivors and survivors for length of ICU stay. The patients who stayed longer than 15 days had a higher APACHE II score compared with patients with shorter ICU stay. It was reported that length of stay in the ICU did not have an independent relationship with in-hospital and long-term mortality, compared with other risk factors such as age, comorbidities, diagnosis, and severity of acute illness. The results suggested that most significant short- and long-term effects of the physiological insult of a critical illness occur within the first 10 days of the onset of critical illness [30]. On the other hand, Higgins et al. [3] demonstrated that clinical characteristics such as age, comorbid diseases and APACHE II score did not affect the length of stay. Studies demonstrated that the effect of length of stay in the ICU on long-term mortality was small when compared with other risk factors [30-35].
In the present study, the observed mortality was higher than the predicted mortality. It could be explained by the physiologic and demographic characteristics of the patients at admission, patient age, diagnosis, specific variations in quality of care, and hospital discharge practice. There were several limitations in our study. First, the ICU population may be different in prevalence of chronic disease and in rates of diseases that require ICU care in groups underrepresented in our population. Second, as our purpose was to evaluate variations in actual ICU use, we did not evaluate the appropriateness of admission and discharge guidelines of ICU patients. Third, differences in the factors of technology availability, training activity, staff workload and resource limitations could be important. In Turkey, all of the ICUs are staffed by doctors. There may be considerable variation between and within ICUs in the experience, clinical management and training of the ICU staff.

In conclusion, early identification of patients at risk, both before admission and after discharge from the ICU, may allow some of the physiologic abnormalities contributing to the APACHE II score to be prevented. When the high performance ICUs in which the scoring systems developed were considered, the scores were found higher in ICUs which did not have the same technical facilities. Finally, our findings may represent the practice of institutions in our country. To a large extent, our data demonstrating variation in the relative rates of ICU care would give an interpretation of the Turkish population.

\section{Acknowledgments}

The authors acknowledge the audit committee, who checked all data entered, resolved data inconsistencies, and trained their medical staff collecting the data, including 690 in total ICUs and 330 in total medical staff.

\section{Conflict of interest}

The authors declare no conflict of interest.

\section{References}

1. Goldhill DR, Sumner A. Outcome of intensive care patients in a group of British Intensive Care Units. Crit Care Med 1998; 26: 1337-45.

2. Wunsch H, Brady AR, Rowan K. Impact of exclusion criteria on case mix, outcome, and length of stay for the severity of disease scoring methods in common use in critical care. J Crit Care 2004; 19: 67-74.

3. Higgins TL, McGee WT, Steingrub JS, Rapoport J, Lemeshow S, Teres D. Early indicators of prolonged intensive care unit stay: impact of illness severity, physician staffing, and pre-intensive care unit length of stay. Crit Care Med 2003; 31: 45-51.

4. Iapichino G, Gattinoni L, Radrizzani D, Simini B, Bertolini G, Ferla L. Volume of activity and occupancy rate in in- 
tensive care units. Association with mortality. Intensive Care Med 2004; 30: 290-7.

5. Kajdacsy-Balla Amaral A, Andrade F, Moreno R, Artigas $A$, Cantraine F, Vincent J. Use of the sequential organ failure assessment score as a severity score. Intensive Care Med 2005; 31: 243-9.

6. Vincent J, Ferreira F, Moreno R. Scoring systems for assessing organ dysfunction and survival. Crit Care Clin 2000; 16: 353-66.

7. Vincent JL, Moreno R, Takala J, et al. The SOFA (Sepsis-relate Organ Failure Assessment) score to describe organ dysfunction/failure: on behalf of the Working Group on Sepsis-Related Problems of the European Society of Intensive Care Medicine. Intensive Care Med 1996; 22 707-10.

8. Knaus WA, Zimmerman JE, Wagner DP, Draper EA, Lawrence $D E$. APACHE-acute physiology and chronic health evaluations: a physiologically based classification system. Crit Care Med 1981; 9: 591-7.

9. Wenner JB, Norena M, Khan N, et al. Reliability of intensive care unit admitting and comorbid diagnoses, race, elements of Acute Physiology and Chronic Health Evaluation II score, and predicted probability of mortality in an electronic intensive care unit database. J Crit Care 2009; 24: 401-7.

10. Ho K. Combining sequential organ failure assessment (SOFA) score with acute physiology and chronic health evaluation (APACHE) II score to predict hospital mortality of critically ill patients. Anaesth Intensive Care 2007; 35: 515-21.

11. Levin PD, Sprung CL. The process of intensive care triage. Intensive Care Med 2001; 27: 1441-5.

12. Beck DH, Smith GB, Pappachan JV, Millar B. External validation of the SAPS II, APACHE II and APACHE III prognostic models in South England: a multicentre study. Intensive Care Med 2003; 29: 249-56.

13. Goldhill DR, Withington PS. The effect of case mix adjustment on mortality as predicted by APACHE II. Intensive Care Med 1996; 22: 415-9.

14. Markgraf R, Deutschinoff G, Pientka L, Scholten T, Lorenz $C$. Performance of the score systems Acute Physiology and Chronic Health Evaluation II and III at an interdisciplinary intensive care unit after customization. Crit Care 2001; 5: 31-6.

15. Pettilä V, Pettilä M, Sarna S, Voutilainen P, Takkunen O. Comparison of multiple organ dysfunction scores in the prediction of hospital mortality in the critically ill. Crit Care Med 2002; 30: 1705-11.

16. Ouanes I, Schwebel C, Français A, et al. A model to predict short-term death or readmission after intensive care unit discharge. J Crit Care 2012; 27: 422-6.

17. Kumar A, Aronow WS, Alexa M, et al. Prevalence of use of advance directives, health care proxy, legal guardian, and living will in 512 patients hospitalized in a cardiac care unit/intensive care unit in 2 community hospitals. Arch Med Sci 2010; 30: 188-91.

18. Hantke M, Holzer K, Thöne S, Schmandra T, Hanisch E. The SOFA score in evaluating septic illnesses. Correlations with the MOD and APACHE II score. Chirurg 2000; 71: 1270-6.

19. Metnitz PG, Lang T, Vesely H, Valetin A, Le Gal JR. Ratios of observed to expected mortality are affected by differences in case mix and quality of care. Intensive Care Med 2000; 26: 1466-72

20. Harrison DA, Brady AR, Parry GJ, Carpenter JR, Rowan K. Recalibration of risk prediction models in a large multicenter cohort of admissions to adult, general critical care units in the United Kingdom. Crit Care Med 2006; 34: 1378-88.

21. Vincent JL, Marshall JC, Namendys-Silva SA, et al. Assessment of the worldwide burden of critical illness: the intensive care over nations (ICON) audit. Lancet Respir Med 2014; 2: 380-6.

22. Guo Q, Li HY, Li YM, et al. Compliance with severe sepsis bundles and its effect on patient outcomes of severe community-acquired pneumonia in a limited resources country. Arch Med Sci 2014; 10: 970-8.

23. Colpan A, Akinci E, Erbay A, Balaban N, Bodur H. Evaluation of risk factors for mortality in intensive care units: a prospective study from a referral hospital in Turkey. Am J Infect Control 2005; 33: 42-7.

24. Knaus WA, Draper EA, Wagner DP, Zimmerman JE. An evaluation of outcome from intensive care in major medical centers. Ann Intern Med 1986; 104: 410-8.

25. Rocker G, Cook D, Sjokvist P, Weaver B, Finfer S, McDonald $\mathrm{E}$. Clinician predictions of intensive care unit mortality. Crit Care Med 2004; 32: 1149-54.

26. Juneja D, Nasa P, Singh O, Javeri Y, Uniyal B, Dang R. Clinical profile, intensive care unit course, and outcome of patients admitted in intensive care unit with dengue. J Crit Care 2011; 26: 449-52.

27. Bastos PG, Knaus WA, Zimmerman JE. The importance of technology for achieving superior outcomes from intensive care. Intensive Care Med 1996; 22: 664-9.

28. Vincent JL, Carvalho F. Severity of illness. Semin Respir Crit Care Med 2010; 31: 31-8.

29. Khanagavi J, Gupta T, Aronow WS, et al. Hyperkalemia among hospitalized patients and association between duration of hyperkalemia and outcomes. Arch Med Sci 2014; 10: 251-7.

30. Williams TA, Ho KM, Dobb GJ, Finn JC, Knuiman $M$, Webb SA. Effect of length of stay in intensive care unit on hospital and long-term mortality of critically ill adult patients. Br J Anaesth 2010; 104: 459-64.

31. Piffaretti G, Mariscalco G, Riva F, Fontana F, Carrafiello G, Castelli P. Abdominal aortic aneurysm repair: longterm follow-up of endovascular versus open repair. Arch Med Sci 2014; 10: 273-82.

32. Paul E, Bailey M, Pilcher D. Risk prediction of hospital mortality for adult patients admitted to Australian and New Zealand intensive care units: development and validation of the Australian and New Zealand Risk of Death model. J Crit Care 2013; 28: 935-41.

33. Hogendorf P, Durczyński A, Kumor A, Strzelczyk J. Pancreatic head carcinoma and vascular endothelial growth factor (VEGF-A) concentration in portal blood: its association with cancer grade, tumor size and probably poor prognosis. Arch Med Sci 2014; 10 : 288-93.

34. Thuluvath PJ, Wagennar RR, Verma S. Gender and ethnic differences in the post-liver transplant outcomes of patients with autoimmune hepatitis with acute liver failure at initial presentation: a case-control study. Arch Med Sci 2015; 11: 1227-35.

35. Sharma D, Newman TG, Aronow WS. Lung cancer screening: history, current perspectives, and future directions. Arch Med Sci 2015; 11: 1033-43. 